\title{
Can composition and preheating improve infiltrant characteristics and penetrability in demineralized enamel?
}

\author{
Livia Aguilera GAGLIANONE(a) \\ Carmem Silvia PFEIFER ${ }^{(\mathrm{b})}$ \\ Caroline MATHIAS(a) \\ Regina Maria PUPPIN-RONTANI(c) \\ Giselle Maria MARCHI(a) iD \\ (a) Universidade Estadual de Campinas \\ - Unicamp, Piracicaba Dental School, \\ Department of Restorative Dentistry, \\ Piracicaba, SP, Brazil. \\ (b) Oregon Health \& Science University, School \\ of Dentistry, Division of Biomaterials and \\ Biomechanics, Portland, OR, USA. \\ (c) Universidade Estadual de Campinas \\ - Unicamp, Piracicaba Dental School, \\ Department of Pediatric Dentistry, \\ Piracicaba, SP, Brazil.
}

Declaration of Interests: The authors certify that they have no commercial or associative interest that represents a conflict of interest in connection with the manuscript.

Corresponding Author:

Caroline Mathias

E-mail: caroline.mathias@hotmail.com.br

\section{Introduction}

ht1ps://doi.org/10.1590/1807-3107bor-2020.vol34.0099

Submitted: January 23, 2020

Accepted for publication: June 9, 2020

Last revision: July 6, 2020
Abstract: The composition of infiltrants can influence their physical properties, viscosity and depth of penetration (DP). Strategies are used to increase the DP, such as the addition of diluents or the use of heat. This study aimed to evaluate the effect of preheating and composition on physical properties and DP of infiltrants in demineralized enamel. The groups were assigned, and the following experimental formulations were made: 25\%BisEMA +75\%TEGDMA; 25\%BisEMA +65\%TEGDMA +10\% ethanol; 25\%BisEMA +65\%TEGDMA +10\%HEMA; 100\%TEGDMA; $90 \%$ TEGDMA $+10 \%$ ethanol; 90\%TEGDMA $+10 \%$ HEMA. The samples were photoactivated at two temperatures $\left(25^{\circ} \mathrm{C}\right.$ and $\left.55^{\circ} \mathrm{C}\right)$. Degree of conversion (DC) was performed using an infrared spectrophotometer. Elastic modulus (E), flexural strength (FS) and contact angle (CA) tests were also performed. The DP of an infiltrant in demineralized enamel was determined by confocal laser scanning microscopy (CLSM) using an indirect labeling technique. The data were analyzed by two-way ANOVA and Tukey's test. DC increased after preheating in all the groups; however, 90\%TEGDMA+10\%ethanol showed the lowest DC for both temperatures, and the lowest $E$. Preheating did not influence $E$ or FS. The CA increased at $55^{\circ} \mathrm{C}$ for most groups, but decreased for groups containing HEMA. Temperature did not seem to influence DP, and Icon showed the lowest DP values. The 100\%TEGDMA composition showed more homogeneous penetration, whereas Icon showed heterogeneous and superficial penetration. The preheating technique does not improve all properties in all the material compositions. The composition of a material can influence and improve its properties.

Keywords: Resins, Synthetic; Ethanol; Microscopy, Confocal.

Tooth caries development is a dynamic process that evolves from an imbalance of natural demineralization-remineralization events. ${ }^{1}$ In the incipient pre-cavitation stages, sub-superficial lesions develop, in which the mineral content of the tooth is dissolved and hollow channels are created. These channels increase the porosity of the enamel, which appears clinically as a white spot. ${ }^{1}$ At this stage, the process is still reversible using non-invasive methods, mostly by employing fluoride-based materials, as long as the patient is willing to maintain good oral hygiene and healthy 
dietary habits. ${ }^{2,3}$ If patient compliance is an issue that will ultimately compromise remineralization efforts, another alternative is to stop white spot lesion progression by infiltrating the porosities in the enamel with low-viscosity resin, a so-called infiltrant. This material functions according to capillary forces, which carry resin monomers to the pores of enamel to occlude them. ${ }^{4}$ To this end, the progression of non-cavitated caries lesion in enamel can be avoided by reinforcing the tooth structure. ${ }^{5}$

Different studies have evaluated the penetrability, etching parameters, mode of use and monomeric constitution of infiltrants applied to ensure the sealing of a white spot lesion. ${ }^{3,6}$ The monomeric constitution, surface energy and viscosity of the material can influence the infiltrant penetration depths. ${ }^{6,78}$ The sealing of this lesion by the infiltrant can effectively stop caries progression, because it interrupts the supply of the substrate to bacteria. ${ }^{3}$ However, studies that investigate the use of infiltrants to mask the visual aspect of the white spot at different depths make us reflect on the importance of filling the lesion. How it is filled may influence the aesthetic result of masking. This tells us that it is important to study how the materials perform during penetration of the lesion. ${ }^{9}$ The optical effect that allows the altered enamel to look like the adjacent healthy enamel is related to the similarity between the infiltrating refractive index and the healthy enamel, in comparison with porous enamel, filled with air or water. ${ }^{10,11}$

Thus, the addition of diluents is a strategy that can be used to reduce the viscosity of the infiltrant and to increase the depth of penetration. ${ }^{6}$ However, excess solvent, commonly ethanol or 2-hydroxyethyl methacrylate (HEMA), can jeopardize the physical properties of the infiltrant. ${ }^{12,13}$ Icon commercial infiltrant is composed mainly of triethylene glycol dimethacrylate (TEGDMA), a low viscosity monomer of low-molecular weight. It can undergo hydrolytic degradation, as previously tested in other monomer blends. ${ }^{14}$ Another strategy that can reduce viscosity without resorting to altering the composition is based on heating. This technique has been shown to decrease the viscosity of some restorative composite resins, but it has not been tested on infiltrants, which already have low viscosity. ${ }^{15}$ Therefore, it is reasonable to propose preheating as a way of reducing the viscosity of the infiltrant, regardless of its formulation. Since the increase in the thermal energy of a resin material accelerates the movement of the monomers, the preheating technique could increase the reaction rate, resulting in a higher degree of conversion and improvement of the physical properties of the material. ${ }^{15,16,17}$

To this end, this in vitro study aims to evaluate the influence of composition and preheating of infiltrants on physical properties, degree of conversion, contact angle and depth of penetration in tooth enamel. The null hypotheses tested were: a) The preheating technique would not influence the properties of degree of conversion, contact angle, elastic modulus or flexural strength. b) The composition of the infiltrants would not influence the properties of degree of conversion, contact angle, elastic modulus or flexural strength. c) Depth of penetration in demineralized enamel would not be influenced by the composition or temperature of the tested materials.

\section{Methodology}

\section{Formulation of infiltrants}

Based in a previous pilot study, six experimental formulations of infiltrants and a commercial brand called Icon (DMG, Hamburg, Germany) were tested (Table 1).

The preheated groups used a thermal incubator (ThermoSmart, Astori Tecnica, Poncarale, Italy) to increase the temperature and ensure the samples remained at $55^{\circ} \mathrm{C}$ at the time of photoactivation with an LED light source for $60 \mathrm{~s}$ (Bluephase 5i, Ivoclar Vivadent, Schaan, Liechtenstein, $1000 \mathrm{~mW} / \mathrm{cm}^{2}$ ). The procedure involved pipetting the samples into microtubes positioned in the heating device, and a digital thermometer (BAT 8; Bailey Instruments Inc, Saddle Brook, NJ) inside the samples was used to control the temperature during the experiments. Both the preparation of the experimental infiltrants and the tests were performed at a controlled temperature of $25^{\circ} \mathrm{C}$. Half of the samples were developed at ambient temperature $\left(25^{\circ} \mathrm{C} \pm 1\right)$, and the others were made with material preheated to $55^{\circ} \mathrm{C}$. 
Table 1. Composition (weight percentage) of infiltrants.

\begin{tabular}{lcccccc}
\hline & \multicolumn{5}{c}{ Composition } \\
\cline { 2 - 7 } Infiltrants & BisEMA & TEGDMA & Ethanol & HEMA & CQ & DABE \\
\hline INF & 25 & 75 & 0 & 0 & 0.5 & 1 \\
INF-E & 25 & 65 & 10 & 0 & 10.5 & 1 \\
INF-H & 25 & 65 & 0 & 0 & 0.5 & 1 \\
TEG & 0 & 100 & 0 & 0.5 & 0.5 & 1 \\
TEG-E & 0 & 90 & 0 & 10 & 0.5 & 1 \\
TEG-H & 0 & 90 & & TEGDMA based resin matrix, initiator - additives* &
\end{tabular}

Ingredients were purchased from Sigma-Aldrich (Steinheim, Germany). TEGDMA = triethylene glycol dimethacrylate, BisEMA=ethoxylated bisphenol A glycidyl dimethacrylate, UDMA= diurethane dimethacrylate, $\mathrm{HEMA}=2$-hydroxy-etilmetacrylate, $\mathrm{CQ}=$ camphorquinone,

$\mathrm{DABE}=$ ethyl 4-(dimethylamino)benzoate. ${ }^{*}$ Data provided by DMG, Hamburg.

\section{Degree of conversion}

The degree of conversion (DC) percentage was measured using an infrared spectrophotometer (Nexus 6700 IR Spectrometer, Thermo Scientific, Waltham, MA, USA) in transmission mode. The unpolymerized infiltrant was inserted into silicone rubber molds $(\mathrm{n}=3$; diameter $=11 \mathrm{~mm}$; thickness $=0.8 \mathrm{~mm})$ between two glass slides, and this set was placed on the sample holder of the spectrometer. Spectra were collected before and after photoactivation, and the methacrylate overtone peak at $6165 \mathrm{~cm}^{-1}$ was used to calculate the $\mathrm{DC}^{18}$. The samples were photoactivated using an LED device, for $60 \mathrm{~s}$.

\section{Elastic modulus and flexural strength}

Ten bars $(7 \mathrm{~mm} \times 2 \mathrm{~mm} \times 1 \mathrm{~mm})$ were made using a metal split mold. The size of the sample was adapted to enable single-step polymerization instead of several light-activation steps at different points. ${ }^{19}$ The samples were photoactivated using an LED device, for $60 \mathrm{~s}$ and then stored dry for $24 \mathrm{~h}$ at $37^{\circ} \mathrm{C}$. The three-point bending test was performed with a universal testing machine (Instron, model 4111, Instron, Canton, MA, USA), at $0.5 \mathrm{~mm} / \mathrm{s}$ speed until sample failure. The dimensions of the bars were measured using a digital caliper, and the data were imported into Bluehill software 2 (Instron, Canton, MA, USA), which calculated the elastic modulus $(E)$ in GPa, and flexural strength (FS) in $\mathrm{MPa}$.

\section{Contact angle}

The contact angles (CA) were measured between the glass slide surface and a drop of the infiltrant. Before the test, the surface was cleaned with $100 \%$ ethanol and dried for $24 \mathrm{~h}$ in an incubator at $37^{\circ} \mathrm{C}$. Fresh surfaces were used for each test, using a goniometer equipped with a camera (Digidrop MCAT, GBX, Bourg de Peage, France). Drops of infiltrant (approximately $1 \mu \mathrm{L}$ ) were dispensed on the glass slide using a precision micro syringe. The images were analyzed by a software program (Visiodrop, GBX) that calculates CA $(n=5)$.

\section{Penetration in artificially demineralized enamel}

\section{Preparation of the sample}

The research ethics committee of (xxx) previously approved this study. One hundred human molars were used to prepare the samples, which were extracted at most 3 months prior to testing, and were kept in a $0.5 \%$ chloramine solution ( $\mathrm{pH} 7)$. Roots of the teeth were removed with a cutting machine (Buehler LTD., Lake Bluff, USA). Afterwards, the crowns were cut from the buccal and the lingual surfaces at the mesiodistal axis, and each tooth was split into two halves. The enamel surfaces were flattened using sandpaper with grit sizes \# 600 and \#1200 (Buehler), under water irrigation, and polished using a felt disc and $1 \mu \mathrm{m}$ diamond paste (Buehler). 


\section{Demineralization of enamel}

Fifty-two samples were selected according to initial Knoop microhardness mean values $(319.4 \pm 31.9 \mathrm{KHN})$, obtained by using a microhardness machine (HMV2000; Shimadzu Corporation, Tokyo, Japan), using a Knoop indenter, performing 5 indentations per sample, using a $25 \mathrm{~g}$ load for $10 \mathrm{s.}{ }^{20}$ Afterwards, the surfaces were covered with nail varnish (Colorama, São Paulo, Brazil), leaving a square area of sound enamel exposed ( $5 \mathrm{~mm} \times 5 \mathrm{~mm}$ ). Each sample was kept in $50 \mathrm{~mL}$ of $0.05 \mathrm{M}$ acetate solution $(2.0 \mathrm{~mL}$ of solution $/ \mathrm{mm}^{2}$ of exposed enamel), at $\mathrm{pH} 5$, and at $50 \%$ saturation in relation to the hydroxyapatite, for $10 \mathrm{~h}$ at $37^{\circ} \mathrm{C} .^{20}$

The microhardness was measured again using the same protocol used to determine the initial microhardness, and according to these new measurements. Thirty-six samples with a mean $57.5 \pm 8.1 \mathrm{KHN}$ were selected to determine the depth of penetration. These samples were randomly divided into 12 groups $(n=3)$, each composed of four experimental and one commercial infiltrant plus a control group. A sound enamel sample was used as the positive control (PC) and a demineralized noninfiltered enamel sample, as the negative control $(\mathrm{NC})$, at two temperatures $\left(25^{\circ} \mathrm{C}\right.$ and $\left.55^{\circ} \mathrm{C}\right)$. The 2 experimental infiltrants containing ethanol were excluded from this test due to their unsatisfactory physical properties, according to previous analyses.

\section{Penetration in demineralized enamel and labeling of samples by confocal laser scanning microscopy (CLSM)}

The infiltrant was diffused in the samples using the indirect labeling technique. ${ }^{21}$ The samples were etched with 15\% hydrochloric acid (Icon Etch, DMG) for $120 s^{4}$ and then washed with distilled water for $30 \mathrm{~s}$. Red fluorophore was used for labeling, by keeping the available pores of the samples immersed in an ethanolic solution of $0.1 \%$ Rhodamine B Isothiocyanate (w/v) (RITC, Sigma Aldrich, Steinheim, Germany) $)^{22}$ for $12 \mathrm{~h}$. Afterwards, the samples were air-dried for $10 \mathrm{~s}$ and the infiltrant was applied on the enamel surface. The excess material was removed with cotton pellets after 5 minutes, and the sample was lightcured for $60 \mathrm{~s}$. The excess Rhodamine was removed by keeping the samples in 30\% hydrogen peroxide for $12 \mathrm{~h}$ at $37^{\circ} \mathrm{C}$, and then washing them with distilled water for $60 \mathrm{~s}$.

The nail varnish on the enamel surface was then removed, and the samples were cut into 0.5 -mm-thick slices perpendicular to the enamel surface. Next, the sample slices were polished using sandpaper (1200, 2400, 4000; Buehler). The samples were immersed in a $0.1 \%(\mathrm{w} / \mathrm{w})$ ethanolic solution of $100 \mu \mathrm{M}$ sodium fluorescein (NaFl; Sigma Aldrich) for $3 \mathrm{~min}$, and then washed with deionized water for $10 \mathrm{~s}$.

CLSM images were obtained (Leica, TCS NT; Leica, Heidelberg, Germany) using an objective lens at 20X and 63X magnification, in dual-fluorescence mode, in which RITC and NaFl could be detected simultaneously (RITC: Ex 568 nm, Em 590 nm; NaFl: Ex 488 nm, Em 525/50 nm). Bi-dimensional images were obtained (xyscan), at $1024 \times 1024$ pixel resolution.

The infiltrant depth of penetration was measured at 5 points (both sides from the center of the lesion to the edge of the image, $50 \mu \mathrm{m}$ away from each other), from the surface to the intersection point between the reference line and the deepest extension of red fluorescence, using Image software (NIH, Bethesda, MD, USA). Next, the means were calculated.

\section{Statistical analysis}

The statistical analysis was performed using Minitab software (State College, PA, USA), considering that the data showed normal distribution and homoscedasticity. The results were analyzed with 2-way ANOVA and Tukey's test $(a=5 \%)$.

\section{Results}

\section{Degree of conversion}

The preheating of the infiltrants increased the DC of the materials differently. In the TEG-E group, although the DC increased after preheating, it still had the lowest DC value, indicating that there was an interaction between the factors $(p<0.001$; Table 2$)$. Thus, except for TEG-E, the infiltrants presented a DC between $60-70 \%$ after preheating $(p<0.001)$. Icon presented the highest DC at both temperatures, although it was similar to INF-H after preheating. TEG-E presented the lowest DC at both temperatures $(p<0.001)$. 


\section{Elastic modulus and flexural strength}

There was a significant interaction between the factors (temperature and composition) evaluated for $E(\mathrm{p}<0.001)$ and for FS (p = 0.004) (Table 3). In regard to $E$, the preheating of infiltrants caused no statistical difference among the groups, except for the INF-H and TEG groups. At $25^{\circ} \mathrm{C}$, the INF-H group showed the highest $E$ values, whereas the formulations composed of TEGDMA with ethanol had the lowest values. At $55^{\circ} \mathrm{C}$, the lowest values belonged to the TEG and TEG-E groups. The FS data indicated similar results for the $E$ data, namely that the increase in temperature had no influence on the physical properties of the tested materials.

Table 2. Mean values of degree of conversion (\%) of infiltrants, according to temperature.

\begin{tabular}{|c|c|c|}
\hline \multirow{2}{*}{ Infiltrant } & \multicolumn{2}{|c|}{ DC } \\
\hline & $25^{\circ} \mathrm{C}$ & $55^{\circ} \mathrm{C}$ \\
\hline Icon & $41.3(1.1)^{\mathrm{Ab}}$ & $76.0(1.7)^{\mathrm{Aa}}$ \\
\hline INF & $34.0(1.1)^{\mathrm{Bb}}$ & $61.3(3.8)^{\mathrm{Ca}}$ \\
\hline INF-E & $28.3(1.5)^{\mathrm{Cb}}$ & $62.3(2.5)^{\mathrm{Ca}}$ \\
\hline INF-H & $34.0(2.6)^{\mathrm{Bb}}$ & $73.3(2.1) \mathrm{Aa}$ \\
\hline TEG & $32.7(1.2)^{\mathrm{Bb}}$ & $66.7(1.5) \mathrm{BCa}$ \\
\hline TEG-E & $19.7(3.1)^{\mathrm{Db}}$ & $33.0(3.5)^{\mathrm{Da}}$ \\
\hline TEG-H & $28.7(2.5)^{\mathrm{Cb}}$ & $69.0(2.7)^{\mathrm{Ba}}$ \\
\hline
\end{tabular}

Different letters indicate a statistically significant difference $(p<0.05)$. Uppercase letters compare data in the same column, and lowercase letters compare data in the same line.

\section{Contact angle}

The TEG-E group presented an increase in the contact angle after preheating $(\mathrm{p}<0.001$; Table 4$)$. Icon and TEG groups also showed a rise in the contact angle from $25^{\circ} \mathrm{C}$ to $55^{\circ} \mathrm{C}$, but the most striking rise was detected in the INF group. In addition, among the experimental groups, those containing TEGDMA showed lower CA values at $25^{\circ} \mathrm{C}$. However, the INF-H and TEG-H groups showed a decrease in CA after preheating. Overall, Icon showed the lowest CA, followed by TEG-H at $55^{\circ} \mathrm{C}$, whereas the highest value was observed for the INF group at $55^{\circ} \mathrm{C}$.

\section{Depth of penetration analyzed by CLSM}

A quantitative analysis to detect the depth of penetration of materials (Table 4 ) revealed no statistical differences for temperature $(p=0.449)$ or interactions of the evaluated factors $(p=0.095)$. At $55^{\circ} \mathrm{C}$, the TEG group showed greater depth of penetration compared to Icon and to the other groups. The lowest values of depth of penetration were found for Icon and TEG$H$, regardless of temperature $(p<0.05)$.

The transmission mode shows non-infiltrated enamel pores as dark, and the fluorescence mode show the pores as green, owing to the presence of $\mathrm{NaFl}$. The presence of infiltrant appears in red in the fluorescence mode, and the interaction between the infiltrant material and the lesion body structures can be seen in yellow, in the dual mode.

The positive control (PC) showed sound enamel with no structural alteration, just some defects in the enamel, probably caused during cutting

Table 3. Mean values of elastic modulus (GPa) and flexural strength (MPa) of infiltrants, according to temperature.

\begin{tabular}{lcccc}
\hline \multirow{2}{*}{ Infiltrant } & \multicolumn{2}{c}{$\mathrm{F}$} & \multicolumn{2}{c}{ FS } \\
\cline { 2 - 5 } Icon & $25^{\circ} \mathrm{C}$ & $55^{\circ} \mathrm{C}$ & $25^{\circ} \mathrm{C}$ \\
INF & $1.3(0.2)^{\mathrm{Ba}}$ & $1.4(0.2)^{\mathrm{Aa}}$ & $58.8(11.4)^{\mathrm{CDa}}$ & $62.4(13.1)^{\mathrm{BCa}}$ \\
INF-E & $1.3(0.2)^{\mathrm{Ba}}$ & $1.2(0.2)^{\mathrm{Aa}}$ & $67.3(13.6)^{\mathrm{Ba}}$ & $61.9(12.9)^{\mathrm{BCa}}$ \\
INF-H & $1.3(0.2)^{\mathrm{Ba}}$ & $1.1(0.2)^{\mathrm{Aa}}$ & $59.7(11.1)^{\mathrm{CDa}}$ & $56.2(8.7)^{\mathrm{BCa}}$ \\
TEG & $2.0(0.2)^{\mathrm{Aa}}$ & $1.4(0.2)^{\mathrm{Ab}}$ & $103.2(17.5)^{\mathrm{Aa}}$ & $82.6(10.7)^{\mathrm{Ab}}$ \\
TEG-E & $1.3(0.5)^{\mathrm{Ba}}$ & $0.8(0.2)^{\mathrm{Bb}}$ & $63.5(16.2)^{\mathrm{CDa}}$ & $46.2(14.3)^{\mathrm{Ca}}$ \\
TEG-H & $0.7(0.2)^{\mathrm{Ca}}$ & $0.9(0.2)^{\mathrm{Ba}}$ & $37.2(8.8)^{\mathrm{Ea}}$ & $47.8(10.2)^{\mathrm{Ca}}$ \\
\hline
\end{tabular}

Different letters indicate a statistically significant difference $(p<0.05)$. Uppercase letters compare data in the same column, and lowercase letters compare data in the same line. 
and polishing of the slices, whereas the negative control (NC) confirmed demineralization of the enamel, as indicated by the green porous structures (Figure 1). The ICON group showed a superficial infiltration in the demineralized enamel, with shorter tags among the groups, at $25^{\circ} \mathrm{C}$ (Figure 2, Icon/25). At $55^{\circ} \mathrm{C}$, ICON showed deeper penetration with longer tags than at $25^{\circ} \mathrm{C}$. Preheated ICON

Table 4. Mean values of contact angle (o) and penetration depth $(\mu \mathrm{m})$ of infiltrants, according to temperature.

\begin{tabular}{|c|c|c|c|c|}
\hline \multirow{2}{*}{ Infiltrant } & \multicolumn{2}{|c|}{ Contact angle } & \multicolumn{2}{|c|}{ Depth of penetration } \\
\hline & $25^{\circ} \mathrm{C}$ & $55^{\circ} \mathrm{C}$ & $25^{\circ} \mathrm{C}$ & $55^{\circ} \mathrm{C}$ \\
\hline Icon & $21.9(0.4)^{\mathrm{Eb}}$ & $25.8(0.6)^{\mathrm{Ca}}$ & $30.1(4.8)^{\mathrm{BCa}}$ & $22.3(6.7)^{\mathrm{Ca}}$ \\
\hline INF & $28.1(0.9) \mathrm{Cb}$ & $33.4(0.8)^{A a}$ & $30.2(5.6) \mathrm{BCa}$ & $37.9(6.9) \mathrm{BCa}$ \\
\hline INF-E & $30.4(0.5)^{\mathrm{Ba}}$ & $31.3(2.2)^{A B a}$ & - & - \\
\hline INF-H & $34.1(1.0)^{\mathrm{Aa}}$ & $29.8(0.9)^{\mathrm{Bb}}$ & $43.1(5.7)^{\mathrm{ABa}}$ & $38.3(2.9) \mathrm{Ba}$ \\
\hline TEG & $26.6(0.8) \mathrm{Cb}$ & $28.9(0.7)^{\mathrm{Ba}}$ & $45.5(5.8)^{A B a}$ & 49.7 (1 1.0) Aa \\
\hline TEG-E & $24.1(0.7) \mathrm{Db}$ & $29.3(0.6)^{B a}$ & - & - \\
\hline TEG-H & $28.0(1.6)^{C a}$ & $25.4(1.1)^{\mathrm{Cb}}$ & $31.2(6.3) \mathrm{BCa}$ & $40.7(2.6)^{B a}$ \\
\hline
\end{tabular}

Different letters indicate statistically significant difference $(p<0.05)$. Uppercase letters compare data in the same column, and lowercase letters compare data in the same line.
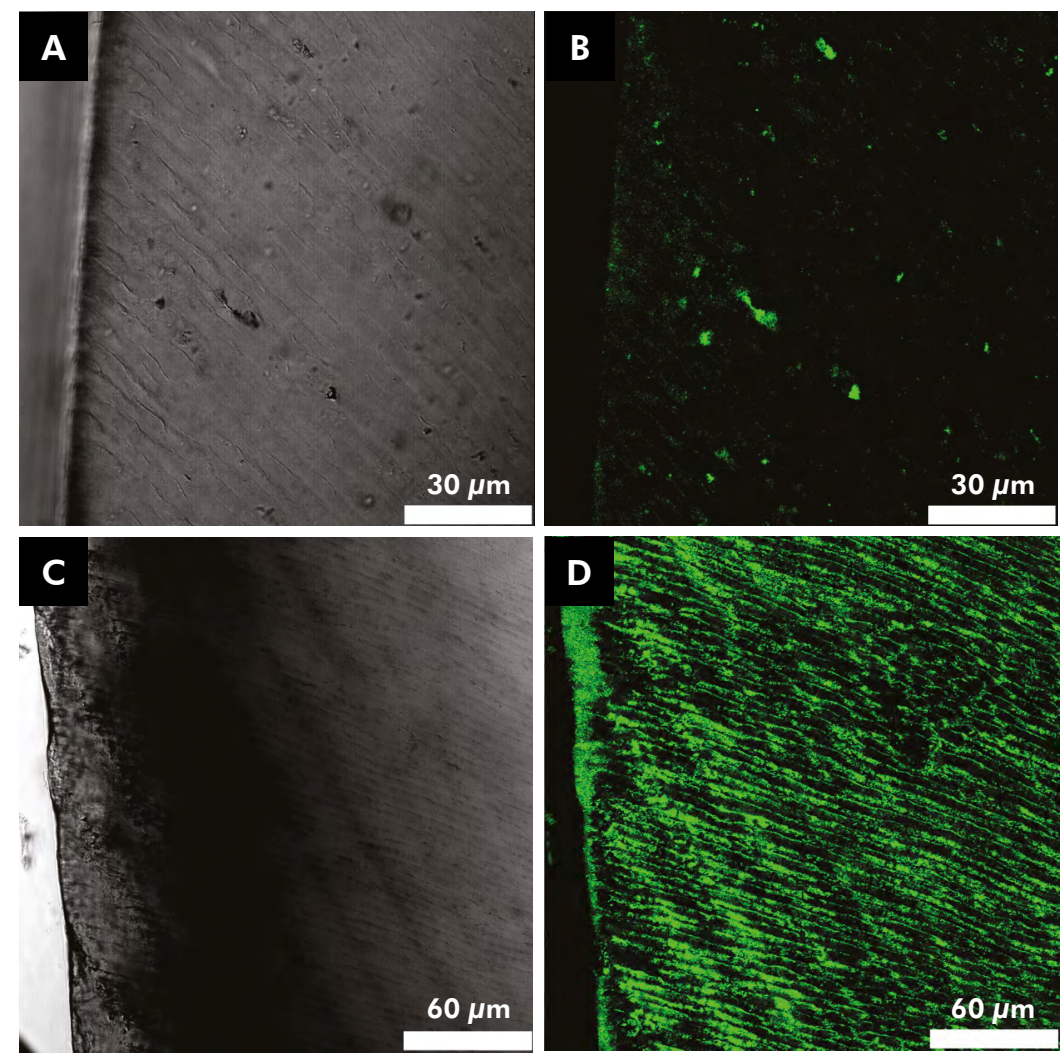

Figure 1. Representative images obtained with CLSM in combined mode of transmission and fluorescence. A. Positive control did not show any structural changes in the enamel (transmission mode). B.- There was no evidence of NaFl in the tissue (fluorescence mode). C. Negative control; porous structures of the lesion body, in black, owing to the scattering of light between the crystals (transmission mode). D. Negative control; green porous structures caused by NaFl, confirming the formation of artificial caries lesions in the enamel (fluorescence mode). 

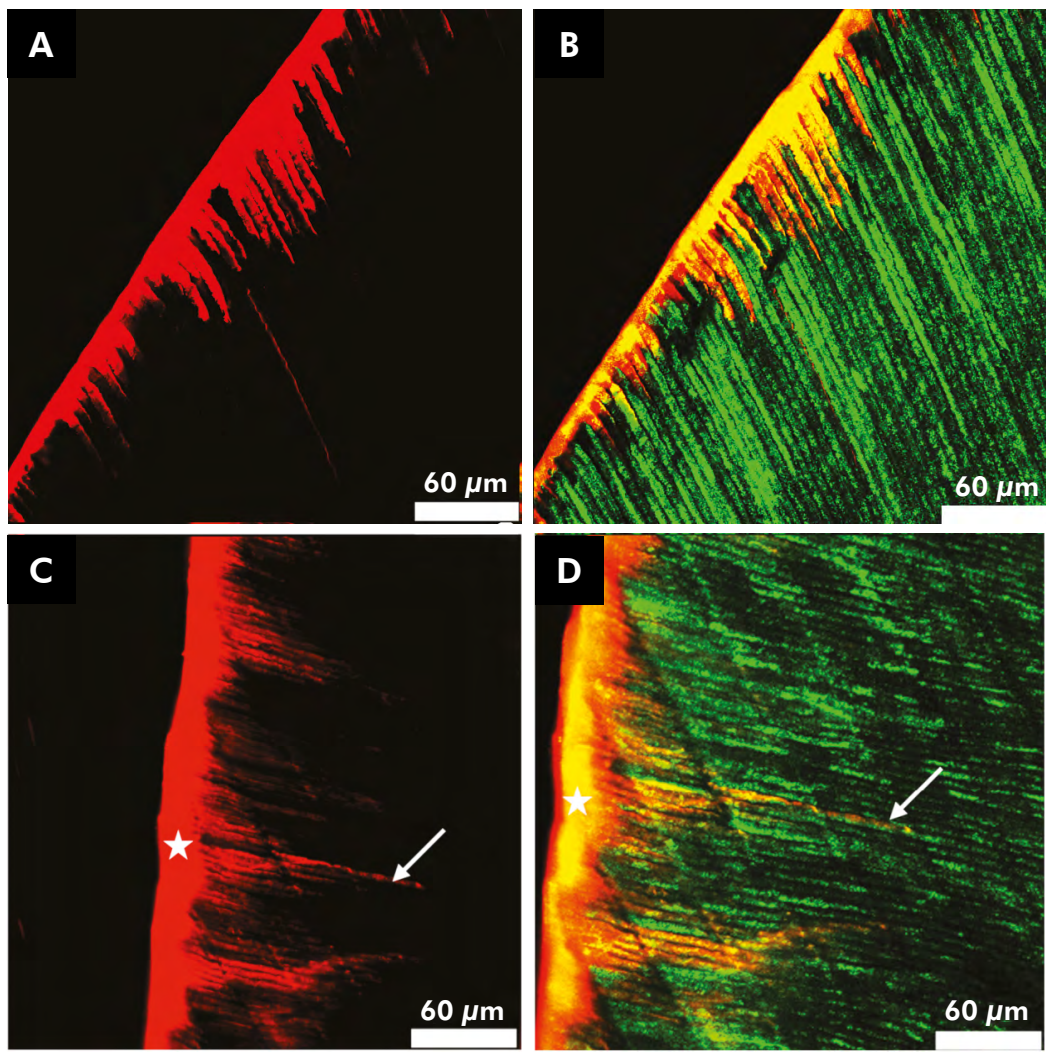

Figure 2. Representative images in dual fluorescence mode: infiltrated structures are in red (RITC), and porous structures are in green ( $\mathrm{NaFl}$. A. Icon at $25^{\circ} \mathrm{C}$ showed superficial penetration. B. Interaction between the infiltrant and the structures of the lesion body can be observed in yellow $\left(25^{\circ} \mathrm{C}\right)$. C, D. Icon, at $55^{\circ} \mathrm{C}$ presented longer tags (white narrow), but a thick layer of material on the surface was observed, evidencing its retention on the surface of the lesion (white star).

presented longer tags, as indicated by the white arrows showing the retention of infiltrant on the surface of the lesions (Figure 2. Icon/55).

The deepest penetration was seen in the TEG group, regardless of temperature, with tags about $45 \mu \mathrm{m}$ inside enamel pores, forming a thick layer of adhesive on the top surface (Figure 3). The preheated group tended to promote more homogeneous infiltration than the TEG group at $25^{\circ} \mathrm{C}$ (Figure 3/TEG55).

\section{Discussion}

The infiltrant composition and increase in temperature modified some of the properties evaluated. The DC was higher for the preheated materials, corroborating other studies on preheating of composite resins. ${ }^{16}$ These studies showed reduced viscosity, increase in DC and improved crosslinking properties, as the major advantages of the preheating method. ${ }^{23,24}$ Thus, the first hypothesis of the study, that preheating would not influence the physical properties of the infiltrants, was rejected. These characteristics pose an advantage, since they increase the resistance of the infiltrant material to water sorption and hydrolytic degradation, and also improve the physical properties of the material. The highest conversion value of $25^{\circ} \mathrm{C}$ was attained by the TEGDMA-based commercial material (Icon) at $41.3 \%$, whereas the experimental groups presented statistically lower values. Thus, it is reasonable to assume that this material has a higher concentration of initiators, and includes additives and other compositional trade secrets. The value found for the Icon DC was close to the values found in other studies, that is, from $50-56 \%$ DC. ${ }^{25,26}$ By comparing the results obtained using the commercial materials with the supposedly experimental analog (100\% TEGDMA)-showing 

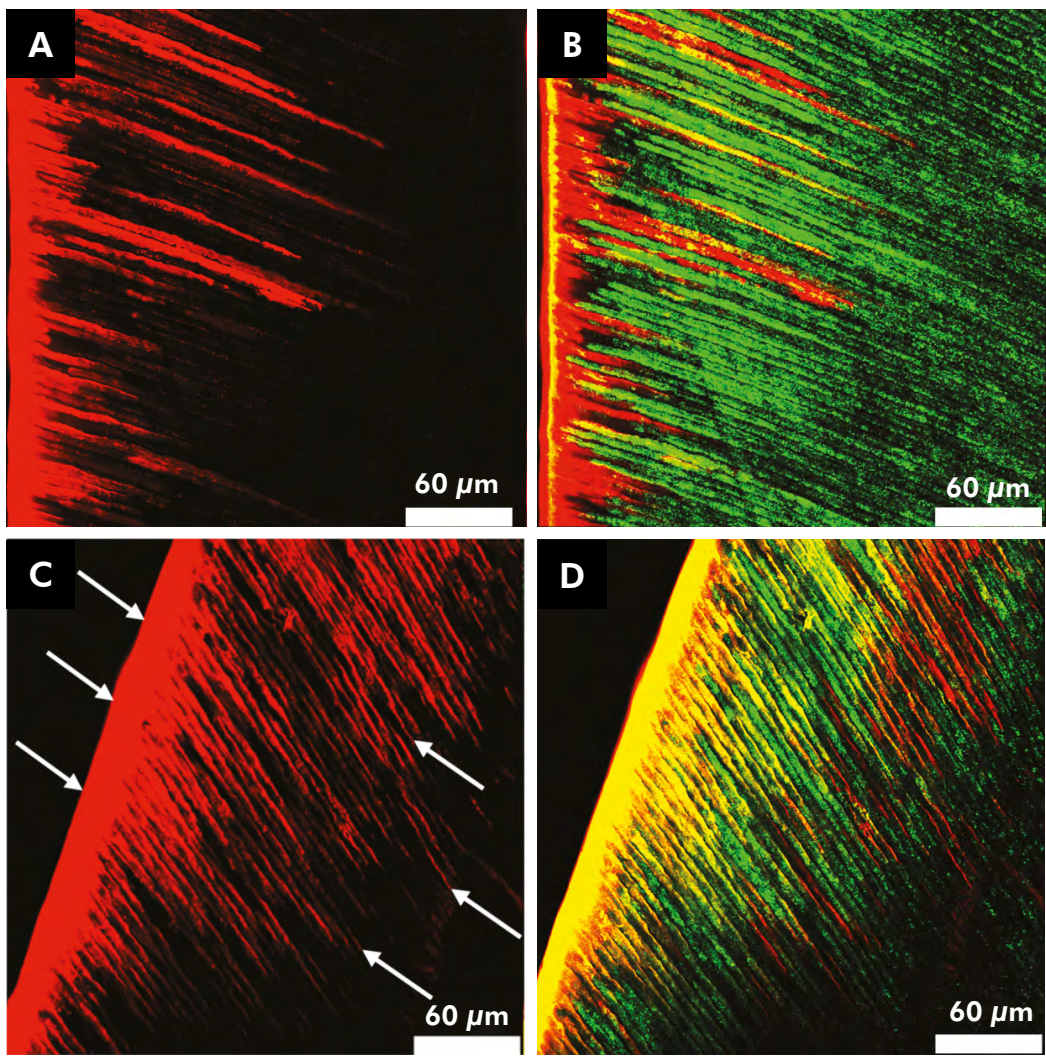

Figure 3. TEGDMA-based group showed deep infiltration into the lesion, regardless of the temperature (dual fluorescence mode). A, B. TEGDMA group at $25^{\circ} \mathrm{C}$. C, D. TEGDMA group at $55^{\circ} \mathrm{C}$, showing that the heated infiltrant presented more homogeneous infiltration than the resin at $25^{\circ} \mathrm{C}$ (white narrow).

just $32.7 \%$ of DC under the same test conditions -it is clear that the remaining experimental groups can be compared only with the experimental control. As expected, raising the temperature led to improved DC, owing to increased molecular mobility and decreased viscosity of the system. ${ }^{27}$ In addition, the frequency of collision of unreacted monomers and radicals is greater when the curing temperature is elevated, if this temperature is below the glass transition temperature. ${ }^{27}$ However, despite the average $30 \%$ increase in conversion for the groups polymerized at $55^{\circ} \mathrm{C}$, the expected improvement in physical properties ${ }^{28}$ was not observed. The results demonstrate that preheating did not influenced the $E$ and FS of most of the groups, corroborating the results of another study on composite resin..$^{13}$ The presence of Bis-EMA and HEMA increased $E$ and FS both preheated to $25^{\circ} \mathrm{C}$, which could explain the better DC values shown by this group versus those containing Bis-EMA and ethanol. Ethanol can solubilize linear chains, resulting in degradation of the physical properties of the material. Thus, the second hypothesis of the study was also rejected, since the different compositions of the infiltrant influenced its properties.

Another strategy to reduce viscosity of materials is to add some sort of solvent or diluent such as ethanol and/or HEMA to the infiltrant, to improve the depth of penetration. ${ }^{2,8}$ However, all the ethanol content cannot be evaporated clinically, nor can the HEMA be removed after penetration. This could compromise the conversion, since HEMA/ ethanol favor the formation of linear chains, and the dilution of a polymeric network under formation, respectively. ${ }^{29}$ Indeed, the results indicated that even small portions of solvents caused reduced DC and $E$. When evaluating the physical performance of infiltrants according to composition, a homogeneous 
behavior pattern can be observed, where the lowest $E$ and FS values were observed in the TEG-E group, whereas INF-H showed the highest values. The addition of solvents to monomeric mixtures can facilitate the formation of microgels close to where the polymerization reaction begins; these microgels reduce the mobility of the radicals, thus compromising the reaction. ${ }^{8}$ These chains show a distribution of heterogeneous mobility, where the higher the solvent concentration, the greater the formation of microgels, and, consequently, the heterogeneity. ${ }^{13,29}$ On the other hand, the material with BisEMA/ TEGDMA/HEMA in its composition had better results. The INF-H group shows a composition of $90 \%$ bi-functional monomers, which are able to form cross-links among the chains, thus reinforcing the polymer and improving its overall properties ${ }^{30}$; this explains the better results achieved. The addition of $25 \%$ BisEMA to TEGDMA seems to have been beneficial, since TEGDMA is a highly hydrophilic material with a greater potential for degradation than BisEMA. ${ }^{3,8,14}$ Hence, the reduction in TEGDMA concentration, and the addition of a low viscosity hydrophobic monomer such as BisEMA, is a valid strategy for potentially creating lower viscosity materials with satisfactory polymeric properties.

The contact angle reflects the interaction of a non-polymerized liquid on a solid surface. This is an important property that should be considered for penetration of resin materials in dental surfaces. Other factors that also influence resin infiltration are surface energy, viscosity of the material, and capillarity forces of the enamel. ${ }^{6}$ The present study sought to evaluate the effect of preheating and different monomeric compositions on the CA of infiltrants. Considering the influence of the surface energy of the object on the wettability of the material, a glass surface was used instead of dental enamel for the purpose of standardization of the test, and thus exclusively evaluating the effect of preheating and composition. In view of the results, it can be seen that both preheating and composition influenced the CA.

Preheating caused only a slight increase in CA in the materials containing only TEGDMA $(100 \%$ TEGDMA and Icon), whereas the increase in CA after preheating in the INF group, was more expressive.
This may be attributed to the higher viscosity of this group, which had BisEMA in its formulation. Although the viscosity was not tested properly in this study, because the composition of the tested groups was known, it may be inferred that the viscosity of the monomers influenced their properties somewhat. In addition, it can be speculated that raising the temperature to $55^{\circ} \mathrm{C}$ may have promoted a structural molecular change in the non-solvent groups, making the interaction of the material with the surface more difficult, and increasing the CA.

In regard to the materials that contained some type of solvent, an increase in CA could be observed after preheating the TEG-E group. This can be explained by the possible evaporation of ethanol when heated. On the other hand, only mixtures containing HEMA showed lower CA on the solid surface after preheating, thereby presenting the best combinations for this property. Preheating did not volatilize HEMA; therefore, its presence made the group less viscous, owing to its low molecular weight. ${ }^{12}$ This factor, combined with molecular agitation due to heating, may have favored greater dispersion.

Moreover, although the samples were kept at $55^{\circ} \mathrm{C}$, a slight drop in temperature may have occurred when the drop was applied to the glass slide, thus influencing the results. In the other tests, a digital thermometer with a probe was used to ensure that the temperature of the samples would be maintained immediately before photoactivation. However, our methodology made this resource inadvisable, since a probe would interfere the in the spread of the material on the glass slide, and thus affect the CA.

As for depth of penetration, the groups containing ethanol were excluded due to the unsatisfactory physical results. Some studies have shown that even though there are advantages to having ethanol in the composition, resins free of solvents are the preferred indication. ${ }^{2,7}$ Regardless of the differences found for $\mathrm{CA}$, the temperature did not influence the results of the depth of penetration, since this property was measured on the glass slide. Most likely, the change in viscosity caused by the increase in temperature was not enough to alter the pattern of penetration. In relation to composition, the divergence in performance 
between the Icon and TEGDMA groups at $55^{\circ} \mathrm{C}$ has already been pointed out. The manufacturer of Icon states that the composition of the infiltrant is basically TEGDMA. However, this group presented a different pattern of diffusion, in comparison with the equivalent experimental group that showed the greatest depth of penetration among the preheated compositions. In contrast, Icon had one of the lowest depths of penetration. This leads us to speculate that Icon's composition of $89.1 \%$ tetraethylene glycol dimethacrylate solvated in ethanol $(9.9 \%)^{31}$ probably led the ethanol to cause a difference. That is, ethanol would be responsible for a heterogeneous pattern of penetration in demineralized enamel. Thus, the third hypothesis of the study was partially rejected, since the different compositions of the infiltrant had an effect on the DP, but the preheating did not influence this property. Future investigations must be conducted to define the best composition and to improve the preheating technique of infiltrants.
Moreover, the viability of clinical application of this technique should be evaluated, since it did not present any advantages in all compositions or improve all the tested properties.

\section{Conclusions}

The preheating of the tested materials influenced most of the properties evaluated, except for depth of penetration. However, the preheating technique did not always improve these properties (E/FS and CA), as seen in relation to the composition of the infiltrant, which influenced all the properties tested. Thus, the preheating technique for infiltrants still cannot be indicated for any specific type of composition.

\section{Acknowledgments}

This study was supported by CNPq (Process: 141335/2011-4) and FAPESP (Processes: 2011/22114-2 and 2012/17679).

\section{References}

1. Meyer-Lueckel H, Paris S, Kielbassa AM. Surface layer erosion of natural caries lesions with phosphoric and hydrochloric acid gels in preparation for resin infiltration. Caries Res. 2007;41(3):223-30. https://doi.org/10.1159/000099323

2. Meyer-Lueckel H, Paris S. Infiltration of natural caries lesions with experimental resins differing in penetration coefficients and ethanol addition. Caries Res. 2010;44(4):408-14. https://doi.org/10.1159/000318223

3. Paris S, Meyer-Lueckel H. Infiltrants inhibit progression of natural caries lesions in vitro. J Dent Res. 2010 Nov;89(11):1276-80. https://doi.org/10.1177/0022034510376040

4. Paris S, Meyer-Lueckel H, Cölfen H, Kielbassa AM. Penetration coefficients of commercially available and experimental composites intended to infiltrate enamel carious lesions. Dent Mater. 2007 Jun;23(6):742-8. https://doi.org/10.1016/i.dental.2006.06.029

5. Torres CR, Rosa PC, Ferreira NS, Borges AB. Effect of caries infiltration technique and fluoride therapy on microhardness of enamel carious lesions. Oper Dent. 2012 Jul-Aug;37(4):363-9. https://doi.org/10.2341/11-070-L

6. Paris S, Meyer-Lueckel H, Cölfen H, Kielbassa AM. Resin infiltration of artificial enamel caries lesions with experimental light curing resins. Dent Mater J. 2007 Jul;26(4):582-8. https://doi.org/10.4012/dmi.26.582

7. Paris S, Soviero VM, Schuch M, Meyer-Lueckel H. Pretreatment of natural caries lesions affects penetration depth of infiltrants in vitro. Clin Oral Investig. 2013 Dec;17(9):2085-9. https://doi.org/10.1007/s00784-012-0909-8

8. Araújo GS, Sfalcin RA, Araújo TG, Alonso RC, Puppin-Rontani RM. Evaluation of polymerization characteristics and penetration into enamel caries lesions of experimental infiltrants. J Dent. 2013 Nov;41(11):1014-9. https://doi.org/10.1016/i.jdent.2013.08.019

9. Abbas BA, Marzouk ES, Zaher AR. Treatment of various degrees of white spot lesions using resin infiltration-in vitro study. Prog Orthod. 2018 Aug;19(1):27. https://doi.org/10.1186/s40510-018-0223-3

10. Kim S, Kim EY, Jeong TS, Kim JW. The evaluation of resin infiltration for masking labial enamel white spot lesions. Int J Paediatr Dent. $2011 \mathrm{Jul} ; 21$ (4):241-8. https://doi.org/10.1111/j.1365-263X.2011.01126.x

11. Paris S, Schwendicke F, Keltsch J, Dörfer C, Meyer-Lueckel H. Masking of white spot lesions by resin infiltration in vitro. J Dent. 2013 Nov;41 Suppl 5:e28-34. https://doi.org/10.1016/i.jdent.2013.04.003

12. Mathias C, Gomes RS, Dressano D, Braga RR, Aguiar FH, Marchi GM. Effect of diphenyliodonium hexafluorophosphate salt on experimental infiltrants containing different diluents. Odontology. 2019 Apr;107(2):202-8.

https://doi.org/10.1007/s10266-018-0391-0 
13. Gonçalves F, Kawano Y, Pfeifer C, Stansbury JW, Braga RR. Influence of BisGMA, TEGDMA, and BisEMA contents on viscosity, conversion, and flexural strength of experimental resins and composites. Eur J Oral Sci. 2009 Aug;117(4):442-6. https://doi.org/10.1111/j.1600-0722.2009.00636.x

14. Brambilla E, lonescu A, Mazzoni A, Cadenaro M, Gagliani M, Ferraroni M, et al. Hydrophilicity of dentin bonding systems influences in vitro Streptococcus mutans biofilm formation. Dent Mater. 2014 Aug;30(8):926-35. https://doi.org/10.1016/i.dental.2014.05.009

15. Lucey S, Lynch CD, Ray NJ, Burke FM, Hannigan A. Effect of pre-heating on the viscosity and microhardness of a resin composite. J Oral Rehabil. 2010 Apr;37(4):278-82. https://doi.org/10.1111/j.1365-2842.2009.02045.x

16. Fróes-Salgado NR, Silva LM, Kawano Y, Francci C, Reis A, Loguercio AD. Composite pre-heating: effects on marginal adaptation, degree of conversion and mechanical properties. Dent Mater. 2010 Sep;26(9):908-14. https://doi.org/10.1016/i.dental.2010.03.023

17. Tauböck TT, Tarle Z, Marovic D, Attin T. Pre-heating of high-viscosity bulk-fill resin composites: effects on shrinkage force and monomer conversion. J Dent. 2015 Nov;43(11):1358-64. https://doi.org/10.1016/i.jdent.2015.07.014

18. Stansbury JW, Dickens SH. Determination of double bond conversion in dental resins by near infrared spectroscopy. Dent Mater. 2001 Jan;17(1):71-9. https://doi.org/10.1016/S0109-5641(00)00062-2

19. Gaglianone LA, Lima AF, Gonçalves LS, Cavalcanti AN, Aguiar FH, Marchi GM. Mechanical properties and degree of conversion of etch-and-rinse and self-etch adhesive systems cured by a quartz tungsten halogen lamp and a light-emitting diode. J Mech Behav Biomed Mater. 2012 Aug;12:139-43. https://doi.org/10.1016/i.jmbbm.2012.01.018

20. Magalhães AC, Moron BM, Comar LP, Wiegand A, Buchalla W, Buzalaf MA. Comparison of cross-sectional hardness and transverse microradiography of artificial carious enamel lesions induced by different demineralising solutions and gels. Caries Res. 2009;43(6):474-83. https://doi.org/10.1159/000264685

21. Paris S, Bitter K, Renz H, Hopfenmuller W, Meyer-Lueckel H. Validation of two dual fluorescence techniques for confocal microscopic visualization of resin penetration into enamel caries lesions. Microsc Res Tech. 2009 Jul;72(7):489-94. https://doi.org/10.1002/jemt.20701

22. D'Alpino PH, Pereira JC, Rueggeberg FA, Svizero NR, Miyake K, Pashley DH. Efficacy of composite surface sealers in sealing cavosurface marginal gaps. J Dent. 2006 Mar;34(3):252-9. https://doi.org/10.1016/i.jdent.2005.06.010

23. Blalock JS, Holmes RG, Rueggeberg FA. Effect of temperature on unpolymerized composite resin film thickness. J Prosthet Dent. 2006 Dec;96(6):424-32. https://doi.org/10.1016/i.prosdent.2006.09.022

24. Uctasli MB, Arisu HD, Lasilla LV, Valittu PK. Effect of preheating on the mechanical properties of resin composites. Eur J Dent. 2008 Oct;2(4):263-8. https://doi.org/10.1055/s-0039-1697390

25. Sfalcin RA, Correr AB, Morbidelli LR, Araújo TG, Feitosa VP, Correr-Sobrinho L, et al. Influence of bioactive particles on the chemical-mechanical properties of experimental enamel resin infiltrants. Clin Oral Investig. 2017 Jul;21(6):2143-51. https://doi.org/10.1007/s00784-016-2005-y

26. Inagaki LT, Alonso RC, Araújo GA, Souza-Junior EJ, Anibal PC, Höfling JF, et al. Effect of monomer blend and chlorhexidine-adding on physical, mechanical and biological properties of experimental infiltrants. Dent Mater. 2016 Dec;32(12):e307-13. https://doi.org/10.1016/i.dental.2016.09.028

27. Lovell LG, Newman SM, Bowman CN. The effects of light intensity, temperature, and comonomer composition on the polymerization behavior of dimethacrylate dental resins. J Dent Res. 1999 Aug;78(8):1469-76. https://doi.org/10.1177/00220345990780081301

28. Collares FM, Ogliari FA, Zanchi CH, Petzhold CL, Piva E, Samuel SM. Influence of 2-hydroxyethyl methacrylate concentration on polymer network of adhesive resin. J Adhes Dent. 2011 Apr;13(2):125-9. https://doi.org/10.3290/i.jad.a18781

29. Ye Q, Spencer $P$, Wang $Y$, Misra A. Relationship of solvent to the photopolymerization process, properties, and structure in model dentin adhesives. J Biomed Mater Res A. 2007 Feb;80(2):342-50. https://doi.org/10.1002/jbm.a.30890

30. Asmussen E, Peutzfeldt A. Influence of selected components on crosslink density in polymer structures. Eur J Oral Sci. 2001 Aug;109(4):282-5. https://doi.org/10.1034/j.1600-0722.2001.00057.x

31. Meyer-Lueckel H, Paris S. Improved resin infiltration of natural caries lesions. J Dent Res. 2008 Dec;87(12):1112-6. https://doi.org/10.1177/154405910808701201 